\title{
Renal Failure in Patients with Liver Cirrhosis: Novel Classifications, Biomarkers, Treatment
}

\author{
Beate Appenrodt Frank Lammert \\ Department of Internal Medicine II, University of Saarland, Homburg/Saar, Germany
}

\section{Keywords}

Acute kidney injury - Acute Kidney Injury Network, AKIN . Albumin - Ascites - Cirrhosis - Hepatorenal syndrome . Neutrophil gelatinase-associated lipocalin, NGAL . Vasoconstrictor

\section{Summary}

Renal failure is a severe complication in patients with liver cirrhosis. It is associated with increased mortality and morbidity. Diagnosis is a challenge because it is mainly based on serum creatinine, which does not seem to be an ideal measure of renal function in cirrhosis. The definition of renal failure in these patients has been changed for optimizing treatment and for improving outcome and prognosis. The new criteria are based on the adapted KDIGO (Kidney Disease: Improving Global Outcomes) staging system. The diagnosis of acute kidney injury (AKI) is based on an absolute increase of serum creatinine of $>0.3 \mathrm{mg} / \mathrm{dl}$ from baseline within $48 \mathrm{~h}$ or an increase of $>50 \%$ from baseline. This means smaller changes in serum creatinine in a shorter time frame which may lead to an early identification of renal failure in cirrhotic patients. The former cirrhotic-specific term hepatorenal syndrome (HRS) is now part of the new diagnostic criteria and is called HRS-AKI. The diagnostic criteria of HRS have changed due to the new criteria for AKI. Due to these criteria for HRS, the medical treatment will be started earlier. First-line treatment for renal AKIHRS is the combination of a vasoconstrictor and albumin. Most data exist for terlipressin, a vasopressin analog, as vasoconstrictor. Besides this medical treatment, there are other options like the placement of a transjugular intrahepatic portosystemic shunt, renal replacement, and artificial extracorporeal liver support systems. However, these alternative treatment options have limitations. Liver transplantation is the treatment of choice for these patients and represents the definitive treatment. Using new biomarkers like urinary neutrophil gelatinase-associated lipocalin or interleukin-18 for renal failure in cirrhosis should help to differentiate the causes of renal failure and provide an indication regarding the prognosis.

(c) 2018 S. Karger GmbH, Freiburg

\section{Introduction}

Renal failure is a frequent complication of patients with liver cirrhosis which is associated with increased mortality and morbidity, occurring in one of every 5 patients with cirrhosis [1]. Renal dysfunction is detected in $20-50 \%$ of patients who are admitted to the hospital [2]. It is one of the most serious complications of decompensated cirrhosis and leads to a rapid progress towards death or to liver transplantation. There are different causes of renal dysfunction. Besides acute kidney injury (AKI), chronic kidney failure induced by comorbidities like diabetes mellitus, arterial hypertension, or specific causes such as immunoglobulin A nephropathy or glomerulopathy is frequent; however, the prevalence is still unknown. Outcome depends on the cause of AKI [3].

Hepatorenal syndrome (HRS) is one kind of AKI and a frequent and serious complication in decompensated cirrhosis. In former understanding, HRS is mainly related to renal vasoconstriction with hypoperfusion due to extended splanchnic vasodilatation [4]. The pathophysiological view on HRS has been modified. Apart from the pathomechanism of renal hypoperfusion due to splanchnic arterial vasodilatation and reduction of cardiac output, proinflammatory processes became additional, important mechanisms in the development of HRS. Recent data suggested that increased levels of circulating proinflammatory cytokines are associated with renal failure in patients with cirrhosis. Therefore, in the revised di-

\section{KARGER}

(C) 2018 S. Karger GmbH, Freiburg
PD Dr. med. Beate Appenrodt Klinik für Innere Medizin II

Universitätsklinikum des Saarlandes

Kirrberger Straße 100, 66424 Homburg, Germany

beate-appenrodt@uks.eu 
agnostic criteria of HRS, spontaneous bacterial peritonitis (SBP) is not an exclusion criterion but rather a trigger event for developing HRS [5]. Thus, an early diagnosis of the cause of AKI is relevant for effective treatment and improved outcome. Using biomarkers like urinary neutrophil gelatinase-associated lipocalin (NGAL) or interleukin-18 (IL-18) for renal failure in cirrhosis could help to differentiate the causes of renal failure and provide an indication regarding the prognosis [6]. In the past, AKI was defined as an increase of the serum creatinine value of more than $1.5 \mathrm{mg} / \mathrm{dl}$. However, the serum creatinine value has its limitations such as underestimation caused by muscle wasting in cirrhotic patients. Therefore, the term and the criteria of HRS and renal failure in cirrhosis were recently modified. The new definition of AKI is based on the known scoring system of the Kidney Disease Improving Global Outcomes (KDIGO) group and is based on an increase of serum creatinine [7].

Specific medical treatment of HRS-AKI, the new term for HRS, is a combination of a vasoconstrictor and albumin. The most evidence exists for the vasoconstrictor terlipressin, a vasopressin analog. Liver transplantation, of course, is the best therapeutic option for patients with HRS-AKI regardless of response to medical treatment. The indication for renal replacement treatment (RRT) should be based on the individual severity of renal failure and cirrhosis. RRT rather seems to be a bridging tool than a treatment with increased prognosis in patients with HRS-AKI. It should be a rescue treatment option and not a first-line choice. Other treatment options should be transjugular intrahepatic portosystemic shunt (TIPS) or extracorporeal liver support systems.

In this review, the new criteria for renal failure and HRS as well as pathophysiology, novel biomarkers, and treatment in these patients with cirrhosis are highlighted.

\section{Pathophysiology of Hepatorenal Syndrome}

The concept that HRS is only a functional injury has changed during the last decade and, thus, the definition of HRS probably needs to be revised. Splanchnic vasodilatation causes systemic vascular vasoconstriction and activation of the renin-angiotensinaldosterone system and the sympathetic nervous system which increases the secretion of vasopressin $[4,8]$. Furthermore, splanchnic vasodilatation exacerbates a hyperdynamic circulation which triggers increased hyperdynamic output followed by decrease in cardiac output, which is known as cirrhotic cardiomyopathy [4]. Another important mechanism in patients with cirrhosis and renal failure becomes more and more obvious, i.e. the systemic proinflammatory response in cirrhotic patients - with and without infections. Patients with cirrhosis show a pathological bacterial translocation from the intestinal lumen to the abdominal lymph nodes. This leads to increased circulating levels of lipopolysaccharide-binding protein and tumor necrosis factor-alpha, which cause splanchnic vasodilatation and renal vasoconstriction due to increased nitric oxide levels $[9,10]$. Bacterial infections are a main cause of HRS, and spontaneous bacterial
Table 1. Novel diagnostic criteria of AKI according to the ICA-AKI consensus

\begin{tabular}{ll}
\hline Stage & Definition \\
\hline 1 & $\begin{array}{l}\text { increase in serum creatinine }>0.3 \mathrm{mg} / \mathrm{dl} \text { or an increase of serum } \\
\text { reatinine }>1.5-\text { to } 2 \text {-fold from baseline }\end{array}$ \\
$1 \mathrm{~A}$ & $\begin{array}{l}\text { serum creatinine }<1.5 \mathrm{mg} / \mathrm{dl} \\
1 \mathrm{~B}\end{array}$ \\
2 & $\begin{array}{l}\text { serum creatinine }>1.5 \mathrm{mg} / \mathrm{dl} \\
\text { increase in serum creatinine }>2 \text { - to } 3 \text {-fold from baseline } \\
\text { increase in serum creatinine }>3 \text {-fold from baseline or initiation of } \\
\text { renal replacement treatment }\end{array}$ \\
\hline
\end{tabular}

$\mathrm{AKI}=$ Acute kidney injury; ICA = International Club of Ascites.

infection is one of the most common triggers for HRS-AKI [3]. Furthermore, patients with decompensated cirrhosis show increased levels of cytokines. In these patients, AKI is a common clinical complication [3].

In summary, HRS is not only influenced by hyperdynamic alterations but is also caused by systemic proinflammatory processes due to pathological bacterial translocation in advanced cirrhosis.

\section{Novel Classification}

Recently, the definition of AKI in cirrhosis was modified according to the Acute Kidney Injury Network (AKIN) criteria and the KDIGO criteria, which are already used for AKI in patients without cirrhosis [11]. The traditional definition of kidney failure in cirrhosis was based on an increased serum creatinine value of more than $1.5 \mathrm{mg} / \mathrm{dl}$. This definition had limitations such as delayed diagnosis because of underestimation of renal function (cirrhotic muscle waste with decreased serum creatinine values) as well as relying on fixed cut-off values instead of the detection of small changes in serum creatinine.

The new diagnostic criteria of AKI are based on small changes in serum creatinine and do not rely on a fixed cut-off value or limit of serum creatinine. Based on the novel classification system, AKI in cirrhosis is defined as an increase in serum creatinine of $\geq 0.3$ $\mathrm{mg} / \mathrm{dl}$ within $48 \mathrm{~h}$, or $50 \%$ or 1.5 -fold from baseline in less than 7 days [5]. The International Club of Ascites (ICA) modified this new definition of AKI in cirrhosis, and a new scoring system of AKI in cirrhosis was designed and called ICA-AKI (table 1). This scoring system is based mainly on the percentage increase of serum creatinine from baseline (initial stage) and the peak value of serum creatinine during hospitalization (peak stage). Both kinds of AKI (initial stage and peak stage) are associated with decreased survival during hospitalization $[5,11]$.

For the new definition, a baseline serum creatinine value is important. Patients mainly develop AKI during hospitalization with a baseline value at admission. In this setting, comparing follow-up serum creatinine and baseline serum creatinine values is possible. There are patients who develop AKI before admission to the hospital, presenting with a high serum creatinine value [2]. In this case, the latest serum creatinine value should be used as the baseline 
value. For these cases, the ICA-AKI criteria defined the baseline serum creatinine as a value from the last 3 months.

AKI is classified into three stages (AKI 1, 2 and 3), depending on changes of serum creatinine. Classification into three stages provides information regarding prognosis. Therefore, the 3-month survival is $42 \%$ in stage 2 and $31 \%$ in stage 3 (table 1 ). In contrast to the known KDIGO scoring system, Stage 1 is divided into Stage $1 \mathrm{~A}$ (serum creatinine $\leq 1.5 \mathrm{mg} / \mathrm{dl}$ ) and Stage $1 \mathrm{~B}$ (serum creatinine > $1.5 \mathrm{mg} / \mathrm{dl}$ ) for better prognosis stratification and for closer monitoring of patients with stage $1 \mathrm{~B}$, similar to patients with AKI stage 2 or 3. Development of acute-on-chronic liver failure is more common in patients with stage $1 \mathrm{~B}$.

In summary, results of several studies showed that the novel scoring system, a modified AKIN classification, is useful for renal failure in patients with cirrhosis due to predicting prognosis earlier and more accurately $[6,12]$.

\section{Types of Acute Kidney Injury}

Depending on the cause of AKI, there are different types which can occur in patients with cirrhosis. A study in 152 patients with cirrhosis showed that $70 \%$ of the patients had AKI, $17 \%$ of the patients had AKI and a chronic kidney disease, and $13 \%$ of the patients had chronic kidney disease alone [13]. Renal failure is divided into prerenal, intrarenal (or intrinsic), postrenal, and HRS: HRS-AKI. In hospitalized patients, the most common cause of AKI is prerenal (approximately $70 \%$ of all cases). This may be resolved by volume expansion. The main cause of intrarenal AKI is acute tubular necrosis (ATN). Postrenal AKI is rare in cirrhotic patients. One of the diagnostic challenges is the discrimination between HRS-AKI and intrinsic AKI such as ATN [13].

\section{Former Definition of Hepatorenal Syndrome}

Formerly, HRS was defined as a functional renal failure caused by splanchnic vasodilatation and consecutive renal vasoconstriction [14]. Furthermore, HRS was divided into type 1 and type 2. Nowadays, the view on HRS has changed. The hypothesis of renal hypoperfusion due to splanchnic arterial vasodilatation and reduction of cardiac output has changed. Inflammation became an important factor in the development of HRS. Recent data suggested that increased levels of circulating proinflammatory cytokines are associated with renal failure in patients with cirrhosis. As mentioned above, in the new diagnostic criteria of HRS, SBP is not an exclusion criteria but rather a trigger event for developing HRS [5] (table 2).

\section{Role of Biomarkers}

Renal failure and AKI are characteristic and serious. Beside HRS-AKI, there are other etiologies than HRS. Diagnosis of the
Table 2. Criteria for HRS-AKI according to the ICA consensus

Liver cirrhosis with ascites

Diagnosis of AKI: increase in serum creatinine $>0.3 \mathrm{mg} / \mathrm{dl}$ within $48 \mathrm{~h}$

No response of diuretic withdrawal after $48 \mathrm{~h}$

No response on plasma volume expansion (albumin $1 \mathrm{~g} / \mathrm{kg}$ of body weight)

No nephrotoxic drugs (e.g. NSAIDs, aminoglycosides)

No proteinuria ( $>500 \mathrm{mg} /$ day)

No microhematuria ( $>50$ RBCs per HPF)

No pathological renal ultrasonography

HRS = Hepatorenal syndrome; AKI = acute kidney injury; ICA = International Club of Ascites.

exact etiology of AKI is essential for treatment and prognosis stratification. Prognosis differs according to AKI etiology. Therefore, AKI due to hypovolemia has the best prognosis, in contrast to HRS-AKI [15]. At the present time, the diagnosis of acute and chronic renal failure is a major challenge and relies only on serum creatinine. Several studies have shown that serum creatinine is not an accurate biomarker of renal injury in patients with cirrhosis [16]. Currently, differential diagnosis relies on classical parameters like proteinuria, urine sediment, renal sodium excretion, and glomerular filtration rate and is based on clinical criteria in patients without cirrhosis. However, these parameters come along with different limitations in patients with cirrhosis. Furthermore, these parameters are not validated in cirrhotic patients, and kidney biopsy is rarely performed in cirrhotic patients with AKI in clinical practice. Recently, novel biomarkers have emerged in this setting. Several biomarkers are described in patients with renal failure without cirrhosis and are proven useful in the differential diagnosis of AKI. More and more studies show the validity of these biomarkers in the differential diagnosis in AKI in cirrhotic patients, which is essential because of different prognosis and management $[17,18]$.

The following new kidney biomarkers in urine are investigated in patients with AKI and cirrhosis [19]:

(1) NGAL;

(2) kidney injury molecule-1 (KIM-1);

(3) IL-18;

(4) liver-type fatty acid-binding protein (L-FABP);

(5) albumin.

NGAL, IL-18, KIM-1, and L-FABP are associated with tubular renal injury, while albumin is associated with glomerular injury. Both kinds of biomarkers indicate structural kidney damage. The biomarkers urinary NGAL, IL-18, and albumin are the most promising and the most validated biomarkers in cirrhotic patients [17]. NGAL, a glycoprotein, could help to determine the cause of AKI in cirrhosis [18]. It is overexpressed in the kidney by impaired kidney epithelia. Because of this mechanism, the NGAL level in the urine rises exponentially as well as earlier than serum creatinine during AKI. Some studies showed the usefulness and validity of urinary NGAL in the differential diagnosis of AKI in cirrhotic patients. These studies demonstrate that the NGAL levels are much higher in patients with ATN than in those with HRS-AKI of prerenal, hy- 


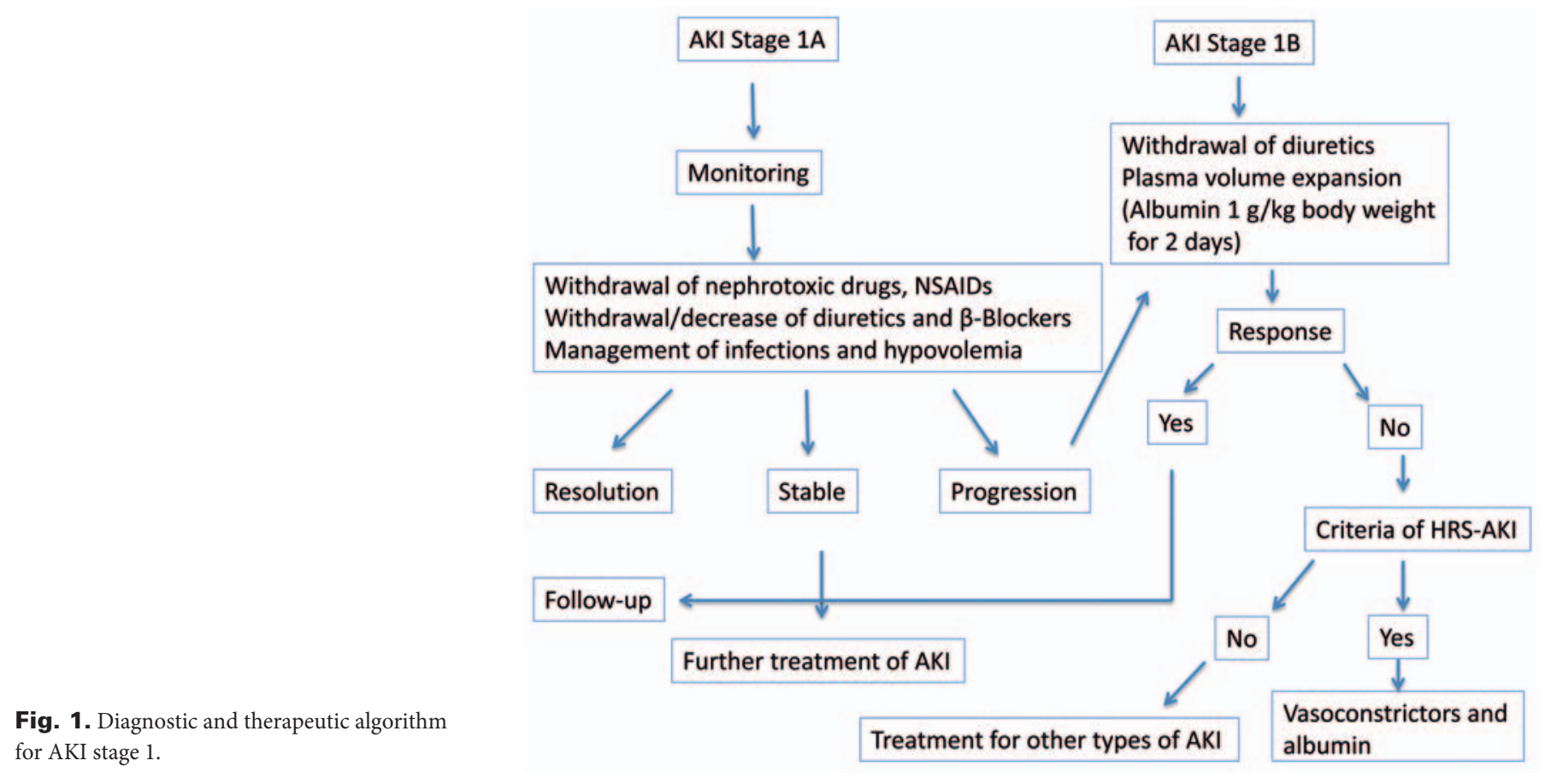

povolemia-induced AKI. The NGAL level in patients with hypovolemia-induced AKI is similar to the NGAL level in patients without AKI. However, there is one limitation of NGAL as a biomarker for AKI: The NGAL level also increases in patients with urinary tract infections because of the production of NGAL in leukocytes [20]. Thus, the level could be false-positive during urinary tract infections.

Another valid biomarker for AKI in cirrhotic patients seems to be IL-18. This biomarker is similar to urinary NGAL and shows the lowest level in patients with hypovolemia-induced AKI and the highest one in AKI due to ATN. A recent meta-analysis analyzed the usefulness of urinary NGAL and IL-18 for prognosing and diagnosing AKI in cirrhotic patients. Both of these discriminated the different causes of AKI with high accuracy [21]. Furthermore, these data showed that higher levels of urinary NGAL and IL-18 were associated with higher short-term mortality [22].

However, there are some overlaps between the different types of AKI and there is no study which compared diagnosis by biomarkers with a reference diagnosis by kidney biopsy. In the future, the combination of different biomarkers could be interesting in the diagnosis of AKI and prognosis in cirrhotic patients with renal failure.

\section{Clinical Management}

The aim of treatment of renal failure in cirrhotic patients is an improvement of kidney function and a bridging to liver transplantation. Early treatment is important and improves the outcome. HRS-AKI should be treated as soon as possible after diagnosis. Then, the kidney function of these patients should be monitored closely. Treatment can be divided into specific and non-specific.
First of all, the administration of nephrotoxic drugs like non-steroidal anti-inflammatory drugs or aminoglycosides should be stopped. Diuretics and/or beta-blockers as well as vasodilators should also be avoided. Correction of triggering factors like infections or hypovolemia is essential. A fluid challenge with intravenous albumin should be started. Volume replacement should be given first for treatment and secondly for the diagnostic approach. $20 \%$ albumin solution at a dosage of $1 \mathrm{~g}$ albumin/kg body weight should be given for 2 days. If renal function does not improve, specific treatment should be started (fig. 1). These recommendations are summarized in the German Guidelines and the European Clinical Practice Guidelines of the European Association of the Study of the Liver [22, 23].

There are different specific options like medical treatment, TIPS placement, renal replacement, extracorporeal liver support, and liver transplantation.

\section{Medical Treatment}

When HRS-AKI is diagnosed, specific treatment should be immediately started. The first choice is the administration of a vasoconstrictive drug in combination with albumin. Vasoconstrictive drugs counteract splanchnic vasodilatation, and renal vasoconstriction decreases with improved renal perfusion [22, 23].

Vasoconstrictors such as vasopressin analogs like terlipressin and alpha-1-agonists like noradrenaline are the most common recommended treatment option. The drug combination with the most existing evidence and data is that of terlipressin and albumin. Data of randomized trials and systematic reviews showed an improvement of kidney function and of survival. The combination of albu- 
min and terlipressin seems to be more effective than a treatment with a vasoconstrictor alone [24]. Albumin increases blood volume and cardiac output, and it is known that decreased cardiac output is associated with HRS. Besides this beneficial volume effect, albumin seems to have anti-inflammatory and antioxidant features with a positive effect on HRS [25]. A recent meta-analysis included five randomized clinical trials and evaluated the effect on terlipressin for type- 1 HRS. Mortality was $64 \%$ in patients who did not receive terlipressin and $48 \%$ in those receiving terlipressin (risk ratio $0.76,95 \%$ confidence interval 0.61-0.95) [26]. However, these response rates were evaluated based on the former definition of HRS. To date, there are no data about the usefulness and effectiveness of the combination terlipressin and albumin in patients with HRSAKI fulfilling the novel modified AKI criteria and not fulfilling the classical criteria of type-1 HRS (patients without a serum creatinine increase $>2.5 \mathrm{mg} / \mathrm{dl}$ ).

Furthermore, the most recent randomized, placebo-controlled, double-blind study, i.e. the REVERSE trial, assessed the efficacy of terlipressin and albumin in rates of response in patients with type-1 HRS [27]. In this study, 196 patients with type-1 HRS were included. Treatment arms were placebo and albumin or terlipressin and albumin. Contrary to other studies, in the REVERSE trial, there was no significantly higher rate of response in the group with terlipressin $(p=0.22)$ and there were no significant differences in short-term survival. In this trial, however, the treatment duration was shorter than recommended, i.e. $30 \%$ of the patients received a treatment for less than 3 days, while $6 \%$ of patients had a treatment duration of 14 days. This seems to be one of the limitations of this study.

\section{Dosage and Application of Terlipressin and Albumin}

Terlipressin should be administered by i.v. bolus with an initial dose of $0.5-1 \mathrm{mg}$ every $4-6 \mathrm{~h}$. The dose can be increased to a maximum of $2 \mathrm{mg}$ every $4-6 \mathrm{~h}$, with a daily maximum of $12 \mathrm{mg}[22,23]$. Improvement of kidney function during treatment is defined as a decrease of serum creatinine of more than $25 \%$ of the baseline value prior to treatment. If kidney function does not improve 3 days after initiation of treatment, the dose of terlipressin should be increased up to $2 \mathrm{mg}$ every $4-6 \mathrm{~h}$.

Alternatively, continuous infusion of terlipressin shows similar response rates with lower adverse effects than administration by bolus $[28,29]$. The initial daily dose of continuous infusion of terlipressin is $2 \mathrm{mg}$. The mean daily dose of terlipressin was significantly higher in the group treated with i.v. bolus administration compared to the group treated with continuous application. Furthermore, the rate of side effects was significantly lower in the group with continuous application of terlipressin (35 vs. 62\%). These data suggest that terlipressin given continuously is effective in lower doses and is tolerated better than i.v. bolus administration. Despite the lower doses of terlipressin in continuous infusion, the effect seems to be more stable by lowering portal pressure permanently.

\section{Albumin}

Albumin solution (20\%) should be administered at a dose of 1 $\mathrm{g} / \mathrm{kg}$ body weight on the first day followed by a dose of 20-40 g per day $[22,23]$. However, the exact dose of albumin in the treatment of type-1 HRS has not been well examined. Some studies suggested that the dose should be adapted to the level of central venous pressure (CVP). Albumin should be stopped if CVP is high and values are above $15 \mathrm{~mm} \mathrm{Hg}$ [30]. In contrast, the validity of CVP in patients with decompensated cirrhosis with an altered cardiac output is limited. Over a longer period, albumin infusion may lead to overload of sodium. Therefore, serum electrolytes should be monitored closely during long-term administration of albumin.

\section{Treatment Duration and Response}

Complete treatment response of type-1 HRS is defined as serum creatinine below $1.5 \mathrm{mg} / \mathrm{dl}$, whereas partial response is defined as a decrease of serum creatinine by $>50 \%$, with a serum creatinine value higher than $1.5 \mathrm{mg} / \mathrm{dl}$. Treatment should be maintained until complete response or for a treatment duration of 14 days irrespective of partial, complete, or non-response. According to the new definition of HRS-AKI, complete response is defined as a final serum creatinine within $0.3 \mathrm{mg} / \mathrm{dl}$ from baseline value, and partial response is defined by regression to final serum creatinine $>0.3 \mathrm{mg} / \mathrm{dl}$ from baseline. In the case of HRS recurrence after complete response and treatment end, the combination should be repeated [22].

\section{Side Effects of Medical Treatment}

Side effects of terlipressin are common because of the potent vasoconstrictive effect. The main side effects are abdominal symptoms like pain or diarrhea and cardiovascular ischemia. When terlipressin is administered by i.v. bolus, the rate of side effects amounts to $40-50 \%$. The rate of discontinuation due to side effects is around $20 \%$ [29]. In the case of severe side effects like cardiovascular events, treatment should be discontinued, otherwise the dose should be decreased and continued under clinical monitoring.

\section{Other Vasoconstrictors}

Besides terlipressin, there are some other vasoconstrictors. In some countries, terlipressin is not available. These vasoconstrictors are octreotide, noradrenaline, and midodrine. For all of these vasoconstrictors, positive studies exist regarding treatment response in HRS. Especially noradrenaline has been proven to be as effective as terlipressin in terms of treatment response and short-time survival $[31,32]$. One randomized controlled study compared terlipressin with noradrenaline as vasoconstrictor in patients with type-1 HRS and showed similar response rates in both treatment groups. There were no differences in frequency and profile of side effects [31]. In a 
recent meta-analysis, these results were confirmed, and noradrenaline seems to be an effective alternative to terlipressin [33]. Noradrenaline is given by continuous infusion at a dose of $0.5-3 \mathrm{mg} / \mathrm{h}$. In contrast to the application of terlipressin, a stay on the intensive care unit during treatment and a central venous line are required. Albumin should be given by means of the usual administration and dosage in combination with the other vasoconstrictors.

The combination midodrine and octreotide with albumin is known in the treatment of type-2 HRS. Furthermore, two studies showed a beneficial effect on renal function and survival in patients with type-1 HRS $[34,35]$. These studies compared the above-mentioned combination with placebo in the treatment of type-1 HRS. In contrast, a randomized study compared the efficacy of midodrine and octreotide versus terlipressin. The response rate in the group treated with terlipressin was significantly higher than in the group treated with midodrine and octreotide (70 vs. $29 \%$ ) [36]. The combination of midodrine and octreotide is used in countries where terlipressin is not yet available. This combination seems to be less effective than terlipressin and albumin, though. However, treatment with terlipressin and albumin in type-2 HRS shows an effective response rate with a high rate of withdrawal. Furthermore, this treatment has the limitation of i.v. administration for outpatients. This combination is not recommended for patients with type-2 HRS [37].

\section{Transjugular Intrahepatic Portosystemic Shunt}

TIPS improves renal function in HRS [38]. However, these data exists mainly for type-2 HRS. Only limited data show a beneficial effect for patients with type-1 HRS [39]. The applicability of TIPS is limited in this clinical setting because of contraindications for TIPS such as severe degree of liver failure. The treatment options and contraindications for TIPS should be evaluated in every patient with type- 1 or type- 2 HRS because it could improve renal function. However, these beneficial effects exists for cirrhotic patients with HRS and ascites. There do not exist any data about the efficacy of TIPS in patients with cirrhosis and AKI without ascites.

\section{Renal Replacement Treatment}

RRT should be considered in the management of HRS-AKI in the case of non-response to medical treatment and should be started in patients with the known indications for RRT such as acid-base or electrolyte imbalance, volume overload, or azotemia. RRT is recommended in patients with cirrhosis who are waiting for liver transplantation [40]. It is still unclear whether RRT improves survival. In contrast, it has been recently observed that critically ill patients with cirrhosis who require RRT have a high mortality independenty of liver transplantation options [41]. The right time for RRT initiation has not been defined in patients with cirrhosis. However, data on AKI in patients with acute liver failure as well as in critically ill patients without liver disease suggest that early RRT improves survival $[42,43]$. Different kinds of replacement treat- ment like hemodialysis or continuous RRT have been used in cirrhotic patients. Continuous replacement seems to be better tolerated by greater cardiovascular stability [43]. In summary, RRT should be used as rescue treatment for HRS.

\section{Liver Support Systems}

There are data from two artificial extracorporeal liver support systems, i.e. the Molecular Adsorbent Recirculating System $\left(\right.$ MARS $^{\circledR}$ ) and the Prometheus ${ }^{\circledR}$ device. The MARS combines continuous RRT and an albumin-enriched dialysate. This device should remove toxins bound to albumin like cytokines and bile acids. In two controlled studies, both of these liver support systems showed promising beneficial effects in patients with type-1 HRS but should be further investigated and the clinical relevance remains still unclear $[44,45]$.

\section{Liver Transplantation}

The best option in patients with renal failure is liver transplantation [46]. All patients with type-1 HRS should be considered for liver transplantation. However, renal failure at the time of liver transplantation has a negative impact on survival after transplantation [47]. Simultaneous liver-kidney transplantation can be indicated in patients with cirrhosis and chronic kidney disease as well as in patients with cirrhosis and sustained AKI irrespective of its type, including HRS-AKI when refractory to drug therapy [48].

\section{Conclusions}

AKI in patients with cirrhosis is a major complication in cirrhosis, and HRS-AKI is one kind of AKI with impaired prognosis. HRS-AKI is mainly related to systemic circulation impairment and inflammatory processes. Recently, the criteria for renal failure and HRS have been modified according to the AKIN criteria. The new definition leads to earlier identification of renal dysfunction, and specific treatment could be initiated sooner. Novel biomarkers like NGAL may be useful for differential diagnosis of the etiology of AKI and may provide an indication concerning the prognosis. The first-line treatment for renal AKI-HRS is the combination of a vasoconstrictor and albumin. For those patients who failed medical treatment, other options like renal replacement or extracorporeal liver support could help to bridge to liver transplantation. TIPS improves survival in patients with HRS-AKI and should be evaluated in these patients. Liver transplantation is the best treatment option for these patients and should be evaluated in patients with renal failure and advanced cirrhosis.

\section{Disclosure Statement}

The authors BA and FL confirm that they do not have any conflicts of interest. 


\section{References}

1 Garcia-Tsao G, Parikh CR, Viola A: Acute kidney injury in cirrhosis. Hepatology 2008;48:2064-2077.

2 Huelin P, Piano S, Solà E, et al: Validation of a staging system for acute kidney injury in patients with cirrhosis and association with acute-on-chronic liver failure. Clin Gastroenterol Hepatol 2017;15:438-445.e5

3 Martín-Llahí M, Guevara M, Torre A, et al: Prognostic importance of the cause of renal failure in patients with cirrhosis. Gastroenterology 2011;140:488-496.

4 Ginès P, Cárdenas A, Arroyo V, Rodés J: Management of cirrhosis and ascites. N Engl J Med 2004;350:16461654.

5 Angeli P, Gines P, Wong F, et al: Diagnosis and management of acute kidney injury in patients with cirrhosis: revised consensus recommendations of the International Club of Ascites. Gut 2015;64:531-537.

6 Belcher JM, Sanyal AJ, Peixoto AJ, Perazella MA, Lim J, Thiessen-Philbrook H, Ansari N, Coca SG, GarciaTsao G, Parikh CR; TRIBE-AKI Consortium: Kidney biomarkers and differential diagnosis of patients with cirrhosis and acute kidney injury. Hepatology 2014;60: 622-632.

7 Fagundes C, Barreto R, Guevara M, Garcia E, Solà E, Rodríguez E, Graupera I, Ariza X, Pereira G, Alfaro I Cárdenas A, Fernández J, Poch E, Ginès P: A modified acute kidney injury classification for diagnosis and risk stratification of impairment of kidney function in cirrhosis. J Hepatol 2013;59:474-481.

$\checkmark$ Schrier RW, Arroyo V, Bernardi M, Epstein M, Henriksen JH, Rodés J: Peripheral arterial vasodilation hypothesis: a proposal for the initiation of renal sodium and water retention in cirrhosis. Hepatology 1988;8: 1151-1157.

9 Wiest R, Lawson M, Geuking M: Pathological bacterial translocation in liver cirrhosis. J Hepatol 2014;60:197209.

10 Bernardi M, Moreau R, Angeli P, Schnabl B, Arroyo V: Mechanisms of decompensation and organ failure in cirrhosis: from peripheral arterial vasodilation to systemic inflammation hypothesis. J Hepatol 2015;63: 1272-1284.

-11 Khwaja A: KDIGO clinical practice guidelines for acute kidney injury. Nephron Clin Pract 2012;120: c179-184.

12 Piano S, Rosi S, Maresio G, Fasolato S, Cavallin M, Romano A, Morando F, Gola E, Frigo AC, Gatta A, Angeli P: Evaluation of the Acute Kidney Injury Network criteria in hospitalized patients with cirrhosis and ascites. J Hepatol 2013;59:482-489.

13 Warner NS, Cuthbert JA, Bhore R, Rockey DC: Acute kidney injury and chronic kidney disease in hospital ized patients with cirrhosis. J Investig Med 2011;59: 1244-1251.

-14 Arroyo V, Ginès P, Gerbes AL, Dudley FJ, Gentilini P, Laffi G, Reynolds TB, Ring-Larsen H, Schölmerich J: Definition and diagnostic criteria of refractory ascites and hepatorenal syndrome in cirrhosis. International Ascites Club. Hepatology 1996;23:164-176.

15 Moreau R, Lebrec D: Diagnosis and treatment of acute renal failure in patients with cirrhosis. Best Pract Res Clin Gastroenterol 2007;21:111-123.

16 Caregaro L, Menon F, Angeli P, Amodio P, Merkel C Bortoluzzi A, Alberino F, Gatta A: Limitations of serum creatinine level and creatinine clearance as filtration markers in cirrhosis. Arch Intern Med 1994, 154:201-205.

17 Qasem AA, Farag SE, Hamed E, Emara M, Bihery A, Pasha H: Urinary biomarkers of acute kidney injury in patients with liver cirrhosis. ISRN Nephrol 2014;2014: 376795.

18 Fagundes C, Pépin MN, Guevara M, Barreto R, Casals G, Solà E, Pereira G, Rodríguez E, Garcia E, Prado V, Poch E, Jiménez W, Fernández J, Arroyo V, Ginès P: Urinary neutrophil gelatinase-associated lipocalin as biomarker in the differential diagnosis of impairment of kidney function in cirrhosis. J Hepatol 2012;57 267-273.
Ariza X, Solà E, Elia C, Barreto R, Moreira R, MoralesRuiz M, Graupera I, Rodríguez E, Huelin P, Solé C, Fernández J, Jiménez W, Arroyo V, Ginès P: Analysis of a urinary biomarker panel for clinical outcomes assessment in cirrhosis. PLoS One 2015; 10:e0128145.

20 Barreto R, Elia C, Solà E, Moreira R, Ariza X, Rodríguez E, Graupera I, Alfaro I, Morales-Ruiz M, Poch E, Guevara M, Fernández J, Jiménez W, Arroyo V, Ginès P: Urinary neutrophil gelatinase-associated lipocalin predicts kidney outcome and death in patients with cirrhosis and bacterial infections. J Hepatol 2014;61:35-42.

21 Puthumana J, Ariza X, Belcher JM, Graupera I, Ginès P, Parikh CR: Urine interleukin 18 and lipocalin 2 are biomarkers of acute tubular necrosis in patients with cirrhosis: a systematic review and meta-analysis. Clin Gastroenterol Hepatol 2017;15:1003-1013.

22 Gerbes AL, Gülberg V, Sauerbruch T, Wiest R, Appen rodt B, Bahr MJ, Dollinger MM, Rössle M, Schepke M: German S 3-guideline 'ascites, spontaneous bacterial peritonitis, hepatorenal syndrome' (Article in German). Z Gastroenterol 2011;49:749-779.

23 Angeli P, Bernardi M, Villanueva C, Francoz C, Mook erjee RP, Trebicka J, Krag A, Laleman W, Gines P EASL Clinical Practice Guidelines for the management of patients with decompensated cirrhosis. J Hepatol 2018;69:406-460.

24 Ortega R, Ginès P, Uriz J, Cárdenas A, Calahorra B, De Las Heras D, Guevara M, Bataller R, Jiménez W, Arroyo $\mathrm{V}$, Rodés J: Terlipressin therapy with and without albumin for patients with hepatorenal syndrome: results of a prospective, nonrandomized study. Hepatology 2002;36:941-948.

25 Chen TA, Tsao YC, Chen A, Lo GH, Lin CK, Yu HC, Cheng LC, Hsu PI, Tsai WL: Effect of intravenous albumin on endotoxin removal, cytokines, and nitric oxide production in patients with cirrhosis and spontaneous bacterial peritonitis. Scand J Gastroenterol 2009;44:619-625.

26 Gluud LL, Christensen K, Christensen E, Krag A: Terlipressin for hepatorenal syndrome. Cochrane Database Syst Rev 2012;(9):CD005162.

27 Sanyal AJ, Boyer TD, Frederick RT, Wong F, Rossaro L, Araya V, Vargas HE, Reddy KR, Pappas SC, Teuber P, Escalante S, Jamil K: Reversal of hepatorenal syndrome type 1 with terlipressin plus albumin vs. placebo plus albumin in a pooled analysis of the OT-0401 and REVERSE randomized clinical studies. Aliment Pharmacol Ther 2017;45:1390-1402.

28 Gerbes AL, Huber E, Gülberg V: Terlipressin for hepatorenal syndrome: continuous infusion as an alternative to i.v. bolus administration. Gastroenterology 2009;137:1179; author reply 1179-1181.

29 Cavallin M, Piano S, Romano A, Fasolato S, Frigo AC, Benetti G, Gola E, Morando F, Stanco M, Rosi S, Sticca A, Cillo U, Angeli P: Terlipressin given by continuous intravenous infusion versus intravenous boluses in the treatment of hepatorenal syndrome: a randomized controlled study. Hepatology 2016;63:983992.

30 Nadim MK, Durand F, Kellum JA, et al: Management of the critically ill patient with cirrhosis: a multidisciplinary perspective. J Hepatol 2016;64:717-735.

31 Singh V, Ghosh S, Singh B, Kumar P, Sharma N, Bhalla A, Sharma AK, Choudhary NS, Chawla Y, Nain CK: Noradrenaline vs. terlipressin in the treatment of hepatorenal syndrome: a randomized study. J Hepatol 2012; 56:1293-1298.

32 Alessandria C, Ottobrelli A, Debernardi-Venon W, Todros L, Cerenzia MT, Martini S, Balzola F, Morgando A, Rizzetto M, Marzano A: Noradrenalin vs terlipressin in patients with hepatorenal syndrome: a prospective, randomized, unblinded, pilot study. J Hepatol 2007;47:499-505.
3 Nassar Junior AP, Farias AQ, D' Albuquerque LA, Carrilho FJ, Malbouisson LM: Terlipressin versus norepinephrine in the treatment of hepatorenal syndrome: a systematic review and meta-analysis. PLoS One 2014; 9:e107466.

34 Angeli P, Volpin R, Gerunda G, Craighero R, Roner P, Merenda R, Amodio P, Sticca A, Caregaro L, MaffeiFaccioli A, Gatta A: Reversal of type 1 hepatorenal syndrome with the administration of midodrine and octreotide. Hepatology 1999;29:1690-1697.

35 Wong F, Pantea L, Sniderman K: Midodrine, octreotide, albumin, and TIPS in selected patients with cirrhosis and type 1 hepatorenal syndrome. Hepatology 2004;40:55-64.

36 Cavallin M, Kamath PS, Merli M, et al: Terlipressin plus albumin versus midodrine and octreotide plus albumin in the treatment of hepatorenal syndrome: a randomized trial. Hepatology 2015;62:567-574.

37 Rodriguez E, Henrique Pereira G, Solà E, Elia C, Barreto R, Pose E, Colmenero J, Fernandez J, Navasa M Arroyo V, Ginès P: Treatment of type 2 hepatorenal syndrome in patients awaiting transplantation: effects on kidney function and transplantation outcomes. Liver Transpl 2015;21:1347-1354.

38 Guevara M, Ginès P, Bandi JC, Gilabert R, Sort P, Jiménez W, Garcia-Pagan JC, Bosch J, Arroyo V, Rodés J: Transjugular intrahepatic portosystemic shunt in hepatorenal syndrome: effects on renal function and vasoactive systems. Hepatology 1998;28:416422

39 Brensing KA, Textor J, Perz J, Schiedermaier P, Raab P, Strunk H, Klehr HU, Kramer HJ, Spengler U, Schild $\mathrm{H}$, Sauerbruch T: Long term outcome after transjugular intrahepatic portosystemic stent-shunt in nontransplant cirrhotics with hepatorenal syndrome: a phase II study. Gut 2000;47:288-295.

40 Thorat A, Jeng LB: Management of renal dysfunction in patients with liver cirrhosis: role of pretransplantation hemodialysis and outcomes after liver transplantation. Semin Vasc Surg 2016;29:227-235.

41 Staufer K, Roedl K, Kivaranovic D, Drolz A, Horvatits T, Rasoul-Rockenschaub S, Zauner C, Trauner M, Fuhrmann V: Renal replacement therapy in critically ill liver cirrhotic patients-outcome and clinical implications. Liver Int 2017;37:843-850.

42 Zarbock A, Kellum JA: Timing of initiation of renal replacement therapy in critically ill patients with acute kidney injury-reply. JAMA 2016;316:1214.

43 Wong LP, Blackley MP, Andreoni KA, Chin H, Falk RJ, Klemmer PJ: Survival of liver transplant candidates with acute renal failure receiving renal replacement therapy. Kidney Int 2005;68:362-370.

44 Bañares R, Nevens F, Larsen FS, et al: Extracorporeal albumin dialysis with the molecular adsorbent recirculating system in acute-on-chronic liver failure: the RELIEF trial. Hepatology 2013;57:1153-1162.

45 Kribben A, Gerken G, Haag S, et al.; HELIOS Study Group: Effects of fractionated plasma separation and adsorption on survival in patients with acute-onchronic liver failure. Gastroenterology 2012;142:782789.e3.

46 Boyer TD, Sanyal AJ, Garcia-Tsao G, Regenstein F, Rossaro L, Appenrodt B, Gülberg V, Sigal S, Bexon AS, Teuber P; Terlipressin Study Group: Impact of liver transplantation on the survival of patients treated for hepatorenal syndrome type 1. Liver Transpl 2011;17: 1328-1332.

47 Gonwa TA, Klintmalm GB, Levy M, Jennings LS, Goldstein RM, Husberg BS: Impact of pretransplan renal function on survival after liver transplantation. Transplantation 1995;59:361-365.

48 Nadim MK, Sung RS, Davis CL, Andreoni KA, Biggins SW, Danovitch GM, Feng S, Friedewald JJ, Hong JC, Kellum JA, Kim WR, Lake JR, Melton LB, Pomfret EA, Saab S, Genyk YS: Simultaneous liver-kidney transplantation summit: current state and future directions. Am J Transplant 2012;12:2901-2908. 